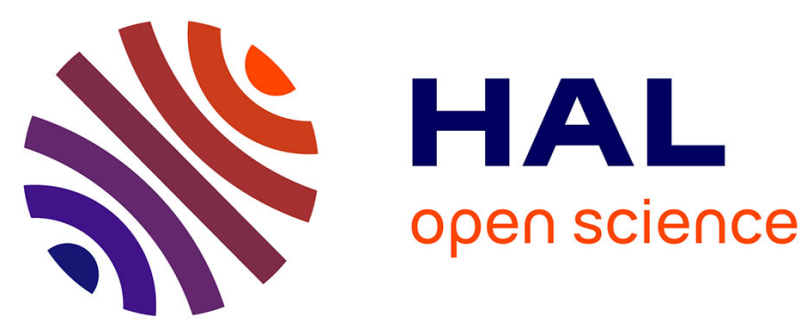

\title{
Probabilistic model for random uncertainties in steady state rolling contact
}

\author{
Luc Chevalier, S. Cloupet, Christian Soize
}

\section{To cite this version:}

Luc Chevalier, S. Cloupet, Christian Soize. Probabilistic model for random uncertainties in steady state rolling contact. Probabilistic model for random uncertainties in steady state rolling contact, 2005, 258 (10), pp.1543-1554. 10.1016/j.wear.2004.11.012 . hal-00686186

\section{HAL Id: hal-00686186 \\ https://hal.science/hal-00686186}

Submitted on 8 Apr 2012

HAL is a multi-disciplinary open access archive for the deposit and dissemination of scientific research documents, whether they are published or not. The documents may come from teaching and research institutions in France or abroad, or from public or private research centers.
L'archive ouverte pluridisciplinaire HAL, est destinée au dépôt et à la diffusion de documents scientifiques de niveau recherche, publiés ou non, émanant des établissements d'enseignement et de recherche français ou étrangers, des laboratoires publics ou privés. 


\title{
Probabilistic model for random uncertainties in steady state rolling contact
}

\author{
Luc Chevalier $^{\mathrm{a}, *}$, Sylvain Cloupet $^{\mathrm{b}}$, Christian Soize $^{\mathrm{a}}$
}

\begin{abstract}
Wear phenomena involve a large number of physical and mechanical parameters which are not always well known or controlled during relative movement between two bodies. Numerous industrial applications necessitate an evaluation of technological component life time and wear modelling often fails to give accurate estimation.

We use the classical Archard's wear model where wear is related to dissipated power. It appears that great dispersion can occur in the estimation of dissipated power related to a lack of knowledge of certain parameters.

We present here a probabilistic approach of the contact problem resolution. We consider the specific contact problem in the case of steady state rolling. A wear apparatus has been used to test different materials and we use the simplified model Fastsim to evaluate slip and tangential traction in the contact zone. For each parameter of the simulation, we construct a probabilistic density function with the only information available.
\end{abstract}

A Monte-Carlo method is implemented and the resolution of numerous cases allows the dissipated energy to be evaluated as a mean value and a confidence region for $95 \%$ viability.

(C) 2004 Elsevier B.V. All rights reserved.

Keywords: Rolling contact; Wear modelling; Probabilistic approach

\section{Introduction}

\subsection{Industrial context and scientific goal}

In the blowing machinery, roller and cam are used to control and synchronise kinematics of different components [1]. This cyclic rolling contact generates surface-wear problems, in particular the transfer can preform. This element conveys preforms from oven to mould where the blowing step takes place (Fig. 1). The injected preforms are griped and transported in mould. The transfer cam governs the grip movement as it is shown in Fig. 1. In industrial condition, the normal load applied on the contact area varies between 250 and $350 \mathrm{~N}$ and the roller velocity is about 2 and $3 \mathrm{~m} / \mathrm{s}$.

\footnotetext{
* Corresponding author. Tel.: +3316095 77 85; fax: +3316095 7799 E-mail address: luc.chevalier@univ-mlv.fr (L. Chevalier).
}

The aim of the global study is to implement a wear simulation software which can predict cam life time. Identification of the wear law parameters is one the scientific goal of the study. A wear apparatus has been realised from which it is possible to managed operational conditions representative of industrial environment. This paper focuses on a probabilistic approach to take into account the experimental uncertainties in the identification process of the wear model.

\subsection{Archard's wear law}

The loss of material due to the cyclic rolling contact loading is modelled by the Archard's law [2]. A similar form is proposed by [3] and the mathematical expression is given by Eq. (1).

$$
W=\frac{K}{H} T L
$$




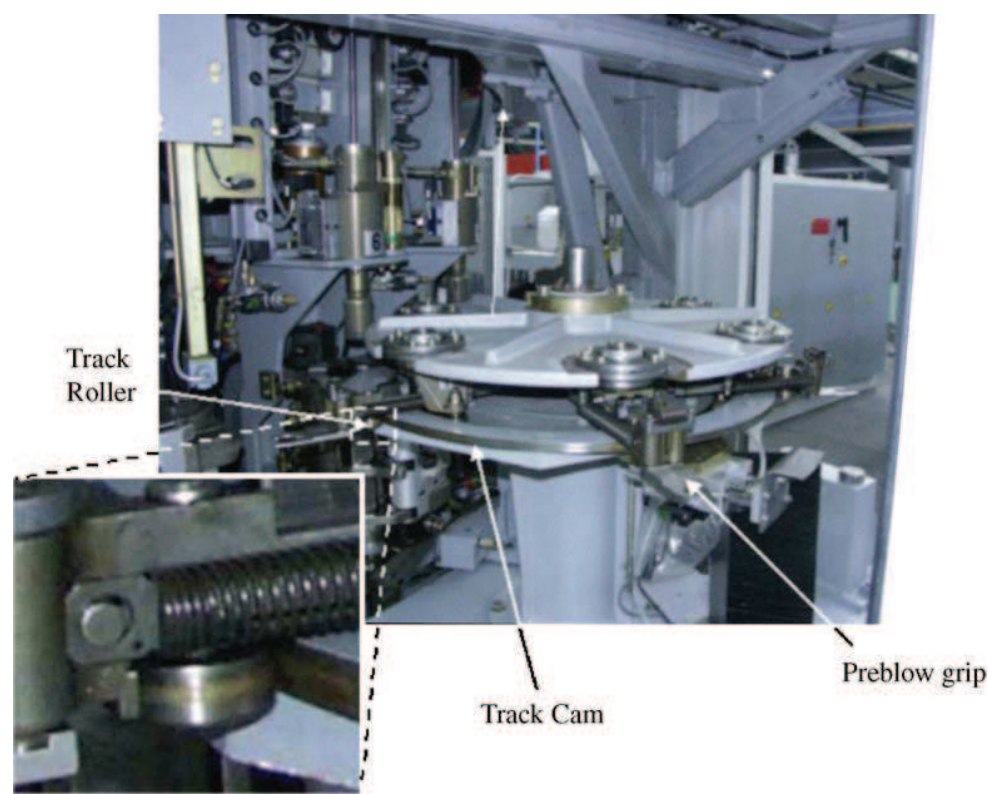

Fig. 1. Photography of the cam for preform transfer.

$W\left(\mathrm{~m}^{3}\right)$ is the volume of wear, $L(\mathrm{~m})$ the sliding length of the abrasive particle, $T(\mathrm{~N})$ the tangential load and $H\left(\mathrm{~N} / \mathrm{m}^{2}\right)$ is the material hardness. In Eq. (1), $K$ is a non-dimensional coefficient that characterised a couple of materials. Magnée gives a microscopic interpretation of the "Archard's factor" $K$ in [4]. Tangential work is calculated by the product $T$ times $L$. In an instantaneous form of this model, the wear rate $(\mathrm{m} / \mathrm{s})$ is directly proportional to the dissipate power $P_{\mathrm{d}}$ as defined in the Eq. (2).

$\dot{W}=\frac{K}{H} P_{\mathrm{d}}$

This energy is not uniformly distributed on the contact area and it is necessary to calculate tangential surface-traction distribution $\tau$ and the sliding velocity $w_{\mathrm{g}}$ at each point on the contact area to specify the distribution of dissipated energy per unit surface. We define the wear depth rate $\dot{u}(\mathrm{~m} / \mathrm{s})$ by Eq. (3).

$\dot{u}=\frac{\mathrm{d} \dot{W}}{\mathrm{~d} S}=\frac{K}{H} \tau w_{\mathrm{g}}$

During a single passage of the roller on the cam track, the increment of the wear depth is obtained by integration over time $(t)$ of depth wear rate from zero to $\Delta t=2 a(y) / V$. $V$ is the rolling velocity and $2 a(y)$ is the length of contact band as shown in Fig. 2. This yields to the incremental wear depth per roller passage $\delta u / \delta n$ given by Eq. (4) where $P_{l}(y)$ is the dissipated power per unit length.

$\frac{\delta u(y)}{\delta n}=\frac{K}{H V} P_{l}(y)$

$P_{l}(\mathrm{y})$ is calculated by integration over $x$ (rolling direction) of tangential traction $\tau(x, y)$ times sliding velocity $w(x, y)$. Those

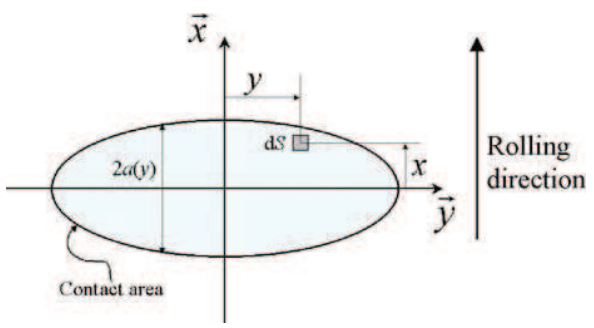

Fig. 2. Elliptic contact area.

quantities are inputs for the wear simulation software. In that way, Eq. (4) form of Archard's model will help to simulate wear profiles evolution.

\section{Wear during rolling contact}

\subsection{Wear apparatus presentation}

An experimental apparatus has been designed to manage wear tests under controlled conditions. Its conception is simple: three rollers are loaded by an elastic mechanism to assure a normal load $N$. The load value is chosen to give a contact area between the steel test cylinder and the roller, representative of the cam-roller industrial problem. The specific steel used is similar to the industrial cam material used for the blowing machinery. Rollers are the same too. Fig. 3 presents the wear apparatus.

The three rollers are oriented at $120^{\circ}$. The same normal load $N$ is managed by each roller. The test cylinder rotation is govern by the traction idler fixed at motor by a belt. Rotation is only imposed at the test cylinder, friction gen- 


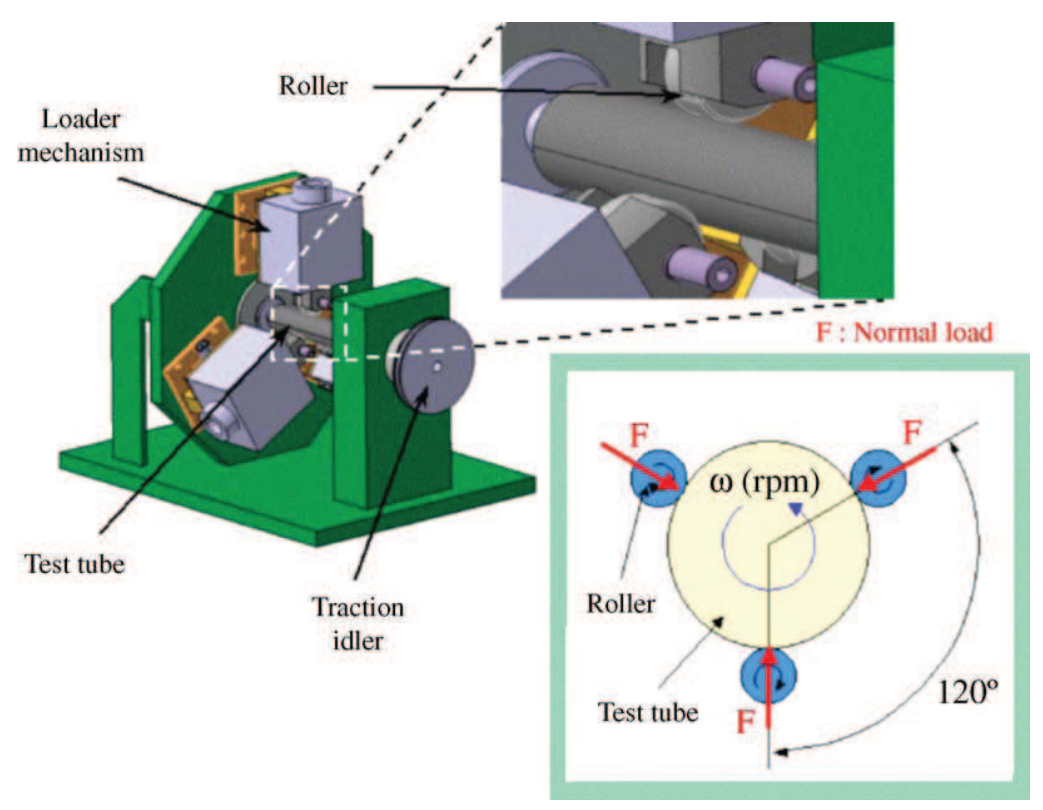

Fig. 3. Presentation of wear apparatus.

erates the roller rotation. Dimensions of cylinder and roller are:

- radius roller $=40 \mathrm{~mm}$,

- radius of crowned outer $=1000 \mathrm{~mm}$,

- radius of test tube $=50 \mathrm{~mm}$.

Wear tests carried out at uniform rotation rate. Problem to solve is then axisymmetric and wear profiles along $y$ direction (cylinder direction) is representative of wear evolution over the all cylinder. Profile measurements are realised with a mechanical profile measurement apparatus at each time step of the test.

The internal slack in roller induces an angular uncertainty $\gamma$ between the rotation axis of the roller and the cylinder axis $\left(\gamma_{\max }=2 / 1000 \mathrm{rad}\right)$. This angular uncertainty between the roller axis and test cylinder axis induces local slips.

\subsection{Uncertainties and fluctuation during wear test}

The loading mechanism has a high stiffness $(k=690 \mathrm{~N} / \mathrm{mm})$. The normal load $N$ is applied with an elastic spring. During the test, the wear depth evolution has a decreasing effect on the normal load. On the other hand, dissipated energy that occurs during the test has an increasing effect on the contact area temperature. This induces an increase of the temperature for the solids involved in the contact loading. The components expansion have an increasing effect on the normal load. Finally, we observe fluctuations of $N$ during a test. These are represented on Fig. 4b. Average load is $307 \mathrm{~N}$ and the standard deviation is $32 \mathrm{~N}$.

\subsection{Uncertainties during wear measurement}

Tests are carried out at various $N$ values. In the following, we will focus on $N$ equal to $300 \mathrm{~N}$ (the one presented Fig. 5) and the rotation rate is equal $1000 \mathrm{rpm}$. At each time step (markers on Fig. 4) wear profile is measured. After $450 \mathrm{~min}$, the wear profiles are presented in Fig. 5 for three tests. We observe two major differences between these three profiles: the maximum depth of wear, the width of wear profile.

One can observe on test 1 , the width of the wear zone is $14 \mathrm{~mm}$ and the maximum depth is $0.028 \mathrm{~mm}$. Test 2 shows a width of $18 \mathrm{~mm}$ and a depth of $0.048 \mathrm{~mm}$. Test 3 is similar to test 2 excluded for the depth which is more important: $0.062 \mathrm{~mm}$. One can also observe a profile asymmetry which is characteristic of angular defect. Parameters (geometrical dimension, normal load, angular positioning of roller with respect to the test cylinder ...) value are the supposed to be the same for each test, but one can observe an important dispersion on experimental results. In the following, we will study the influence of the dispersion measured for the wear test parameters on dissipated energy to give an explanation to the large uncertainty for wear evolution.

\section{Mean model of rolling contact}

In this section, we briefly recall the theoretical basis for the resolution of contact problem during steady state rolling. Considering that the contact area is small in regards of the bodies dimensions, we use the half space approximation. Since Love [5] in 1926 who proposed the analytical solution of concentrated loading on a half infinite elastic body, it 
(a)
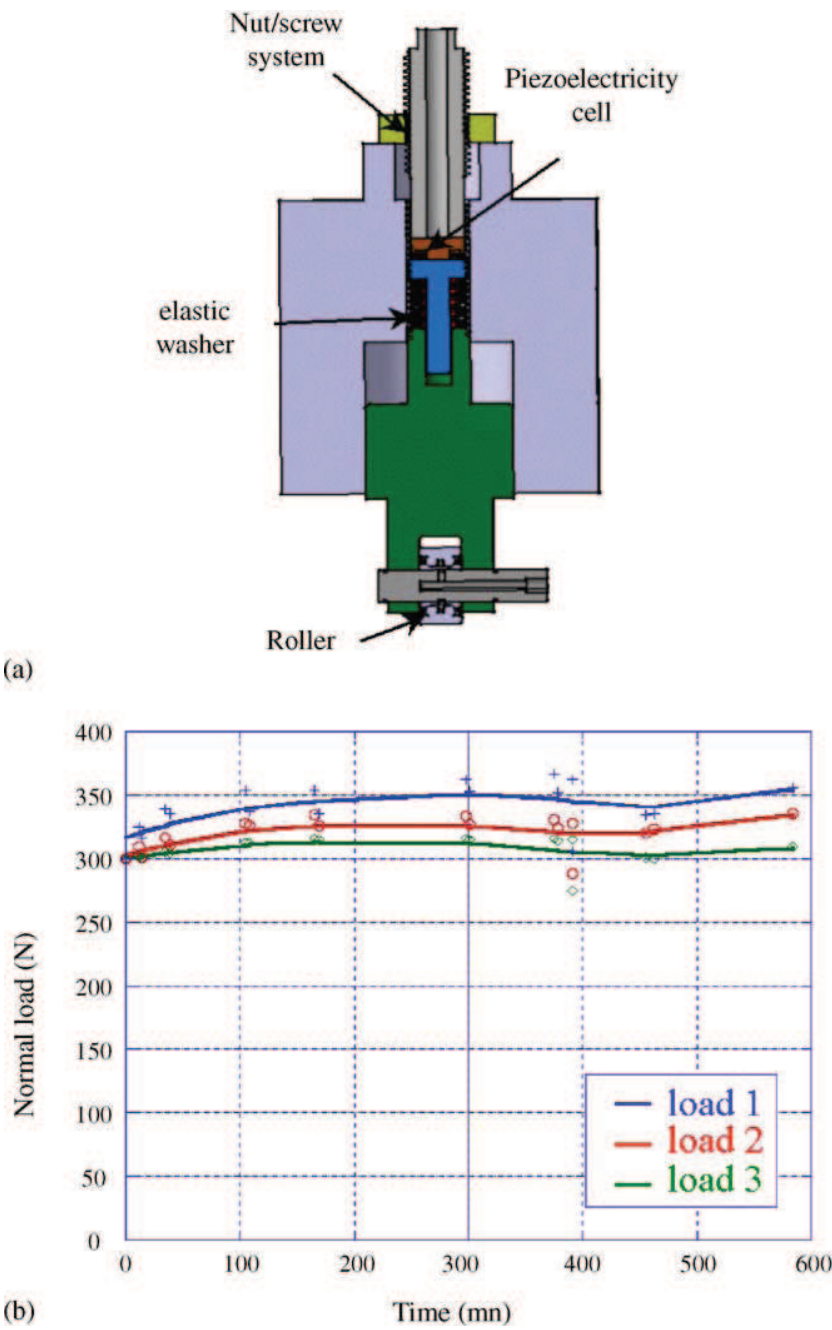

Fig. 4. (a) Loader mechanism, (b) fluctuations of normal load during test.

has been possible to establish relation between normal and tangential traction and the relative displacement in the contact area of two half-infinite bodies (Johnson [6], Kalker [7]).

It has also been established that for two identical bodies (same material elastic constants $E, v$ and $G=E / 2(1+v)$ ) normal and tangential are uncoupled and relative displacements are related to tractions by the relations of Eq. (5).

$$
\begin{aligned}
u(x, y)= & \frac{1}{\pi G} \iint_{\text {contact }}\left(\left\{\frac{1-v}{r}+\frac{v\left(x-x^{\prime}\right)^{2}}{r^{3}}\right\} \tau_{x}\left(x^{\prime}, y^{\prime}\right)\right. \\
& \left.+\frac{v\left(x-x^{\prime}\right)\left(y-y^{\prime}\right)}{r^{3}} \tau_{y}\left(x^{\prime}, y^{\prime}\right)\right) \mathrm{d} x^{\prime} \mathrm{d} y^{\prime} \\
v(x, y)= & \frac{1}{\pi G} \iint_{\text {contact }}\left(\frac{v\left(x-x^{\prime}\right)\left(y-y^{\prime}\right)}{r^{3}} \tau_{x}\left(x^{\prime}, y^{\prime}\right)\right. \\
& \left.+\left\{\frac{1-v}{r}+\frac{v\left(y-y^{\prime}\right)^{2}}{r^{3}}\right\} \tau_{y}\left(x^{\prime}, y^{\prime}\right)\right) \mathrm{d} x^{\prime} \mathrm{d} y^{\prime} \\
w(x, y)= & \frac{1-v}{\pi G} \iint_{\text {contact }} \frac{1-v}{\pi G} \frac{p\left(x^{\prime}-y^{\prime}\right)}{r} \mathrm{~d} x^{\prime} \mathrm{d} y^{\prime}
\end{aligned}
$$

$G$ and $v$ are defined from the elastic coefficient of each body by:

$\frac{1}{G}=\frac{1}{2 G_{1}}+\frac{1}{2 G_{2}} ; \quad \frac{v}{G}=\frac{v_{1}}{2 G_{1}}+\frac{v_{2}}{2 G_{2}}$

The third relation, in case of constant curvature in the contact area, is analogous to the one that Hertz solved and pressure distribution $p(x, y)$ is given to be elliptic. Contact area is and ellipse where half length in $X$ and $Y$ directions are, respectively, denoted $a$ and $b$. The tangential problem is only coupled with the normal problem by the Coulomb friction law [8] recalled on Eq. (7).

$\left\{\begin{array}{l}\vec{w}=\overrightarrow{0} \Rightarrow|\vec{\tau}| \leq \mu p \\ \vec{w} \neq \overrightarrow{0} \Rightarrow|\vec{\tau}|=\mu p \text { and } \vec{\tau}=-\frac{\mu p}{|\vec{w}|} \vec{w}\end{array}\right.$

\subsection{Steady state rolling contact problem}

In the case of steady state rolling, the relative displacement between the two bodies is given by Eq. (8).

$\left\{\begin{array}{l}\frac{w_{x}}{V}=v_{x}-y \phi-\frac{\partial u}{\partial x} \\ \frac{w_{y}}{V}=v_{y}-x \phi-\frac{\partial v}{\partial x}\end{array}\right.$

$v_{x}, v_{y}$ and $\phi$ are the longitudinal and transversal creep coefficients and the spin in the contact area (see Fig. 6), $V$ is the speed of the theoretical contact point when bodies are supposed perfectly rigid.

Assuming that there is no slip on the leading edge of the contact area, one can solve the previous system which leads to Eq. (9).

$$
\left\{\begin{array}{l}
u=(x-a(y))\left(v_{x}-y \phi\right) \\
v=(x-a(y)) v_{y}+\frac{x^{2}-a_{i}^{2}}{2} \phi
\end{array} \text { with } a(y)=a \sqrt{1-\frac{y^{2}}{b^{2}}}\right.
$$

In the linear Kalker theory (when creeps are supposed small or friction ratio extremely high) the above form is valid on the whole contact area and identification with the $u$ and $v$ expressions enable to determine global tangential loading and spin momentum:

$$
\begin{aligned}
& T_{x}=G a b C_{11} v_{x} \\
& T_{y}=G a b C_{22} v_{y}+G(a b)^{3 / 2} C_{23} \phi \\
& M_{z}=G(a b)^{3 / 2} C_{32} v_{y}+G a b C_{33} \phi
\end{aligned}
$$

$C_{i j}$ are given by integrals and only depends of $b / a$ and Poisson ratio $v$. These values are tabulated as $m$ and coefficients of the Hertz problem. When creep values are higher (or when friction is small) tangential traction saturates and becomes equal to $\mu p$. In that case, a numerical resolution must be implemented: this is done in "contact", for example, but leads to prohibitive CPU time when a large number of simulation have to be done. Other authors [9-11], for example, solve 


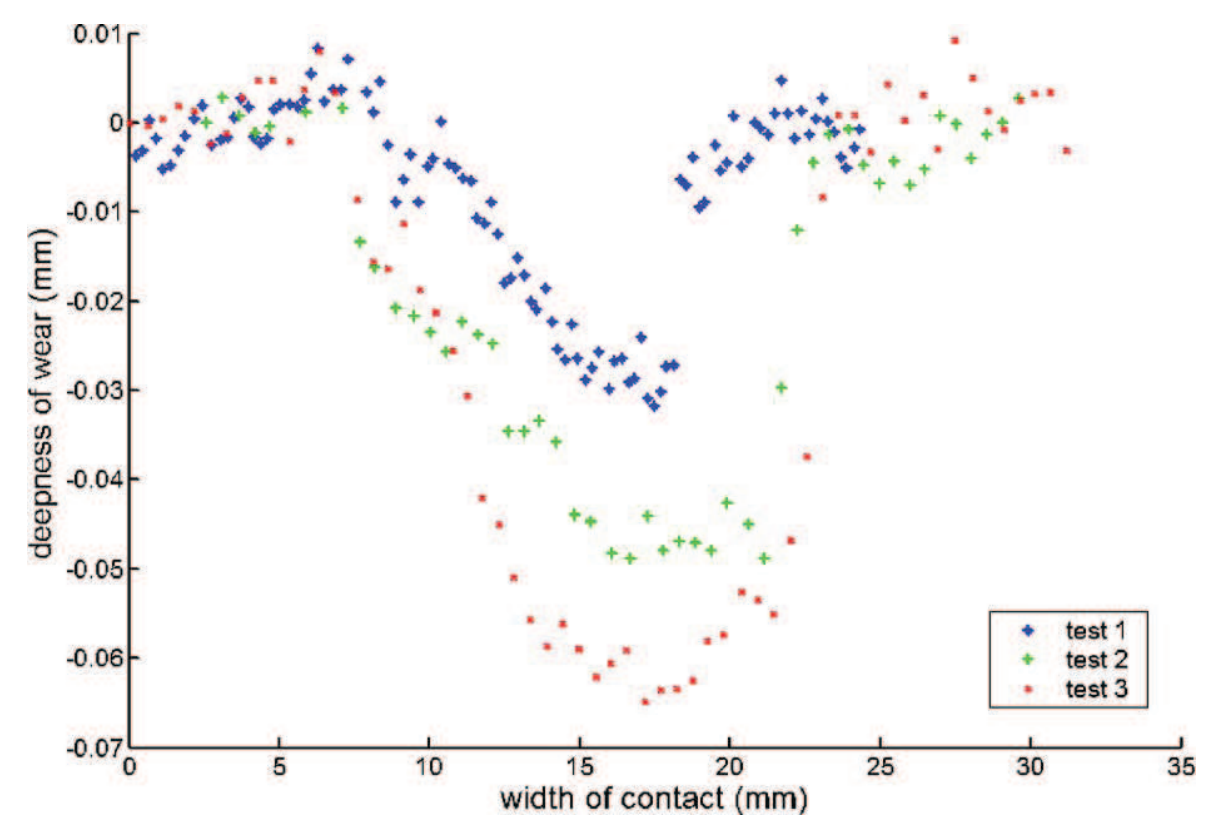

Fig. 5. Wear profile measurements.

the contact problem with various numerical methods but all resolutions give important CPU time. Our choice is to solve an approach but accurate problem proposed by Kalker [7].

\subsection{Kalker approximation of the rolling problem: \\ Fastsim}

An alternative, is to use the Fastsim algorithm also developed by Kalker. Let's examine the proposed simplifications: the local stiffness is modelled as linear relation between tan- gential traction and relative displacements $u$ and $v$ (i.e. $\tau_{x}=L u$ and $\tau_{y}=L v$ ). Solving the above system leads to quasi analytical solution when spin is neglected. The $L$ value depends of $C_{i j}$ coefficient, $G$ shear elastic modulus and ellipse dimensions $a$ and $b$, but we must introduce three values to make sure the $T_{x}$ and $T_{y}$ components of $T L K$ are identical in the case of global adhesion:

$$
L_{1}=\frac{8 a}{3 C_{11} G}, \quad L_{2}=\frac{8 a}{3 C_{22} G}, \quad L_{3}=\frac{\pi a \sqrt{a / b}}{4 C_{23} G}
$$

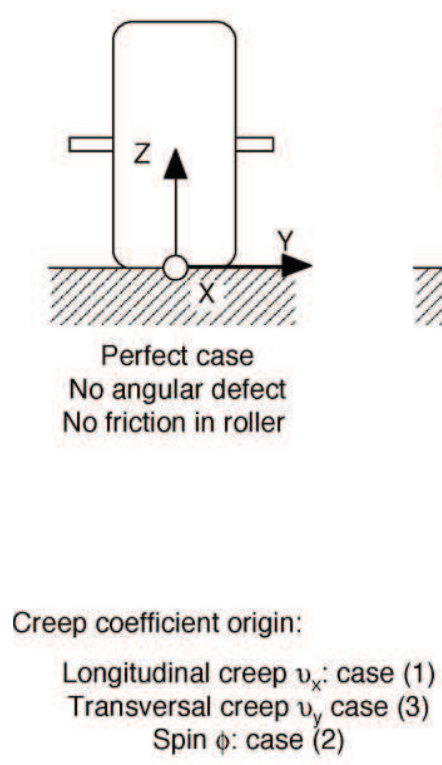

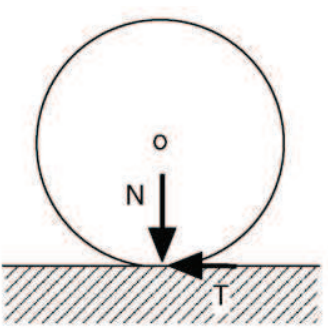

Case (1)

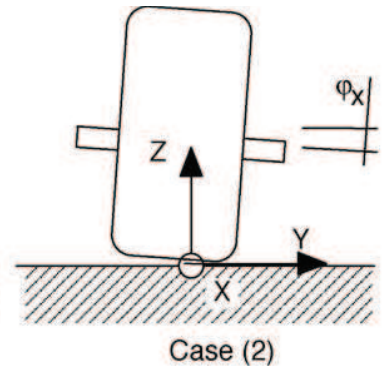

Case (2)

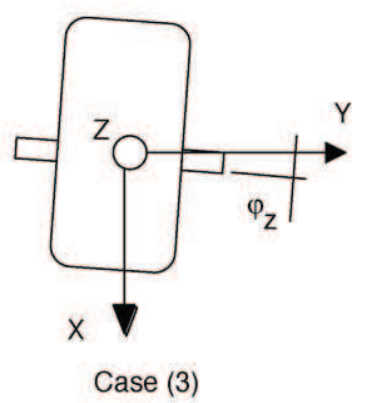

Fig. 6. Position of roller and origin of longitudinal creep (case 1): friction in the roller generates a difference between the two bodies longitudinal velocities; spin (case 2): angular defect $\varphi_{z}$ leads to spin $\phi=\varphi_{z} / R$ where $R$ is the rolling radius of the roller; transversal creep (case 3 ): angular defect $\varphi_{x}$ leads to transversal creep $v_{y}=\varphi_{x}$. 
The simplified problem to solve is expressed by the following relations. It is easy to see that for an elliptic pressure distribution, the slip component will lead to infinity at the rear edge of the contact. This is in contradiction with exact problem simulation and Kalker solves this contradiction by using a parabolic pressure distribution. This is an approximation that increases the maximum pressure $P_{0}$ for $25 \%$ but gives good agreement in the tangential problem.

$\left\{\begin{array}{l}\frac{w_{x}}{V L}=\frac{v_{x}}{L_{1}}-\frac{\phi y}{L_{3}}-\frac{\partial \tau_{x}}{\partial x} \\ \frac{w_{y}}{V L}=\frac{v_{y}}{L_{2}}+\frac{\phi x}{L_{3}}-\frac{\partial \tau_{y}}{\partial x}\end{array}\right.$

The system of Eq. (12) is solved considering traction and slip are equal to zero on the front edge of the contact. This zero value of slip enables tractions to be calculated and one can test if the friction saturation is obtained or not. If it is saturated, friction law is used for tractions calculation and derivation of the pressure expression leads to the slip values. This calculation is managed within the conditions the wear apparatus.

\subsection{Mean problem and results}

We present the results of the steady state rolling contact problem for the case of two steel bodies (Young modulus $E=200,000 \mathrm{MPa}$, Poisson's ratio $v=0.3)$. Under a normal force $N(N=300 \mathrm{~N})$ the Hertz relations gives the pressure distribution on the elliptic contact area (ellipse dimensions $2 a=0.27 \mathrm{~mm}$ and $2 b=3.1 \mathrm{~mm}$ ). In the present case, the aspect ratio of the ellipse is close to 10 ; the small dimension $a$ is in the $V$ direction $(V=\mathrm{m} / \mathrm{s})$.

To solve the tangential problem we consider the mean values of friction coefficient $\mu=0.1$ and longitudinal creep $v_{x}=0.001$. Transversal creep $v_{y}$ and spin $\phi$ mean values are taken to zero. The Fastsim algorithm gives the solution plotted on Fig. 7. It can be seen that a large proportion of the elliptical area is saturated and slip occurs (Fig. 7a-c) shows the traction $\tau_{x}$ distribution and the slip $w_{x}$ assuming pressure distribution is parabolic.

Comparison with the exact solution obtained with 'contact', for example, is sufficiently satisfactory. Consequently we will use Fastsim in the following. It is worth noting that, in special case where $v_{y}=\phi=0$, an analytical solution can be managed which helps the choice of the discretisation of the contact zone in $X$ and $Y$ direction. We choose a $(50 \times 50)$ 2500 elements discretisation. Dissipated power in the contact is equal to $0.073 \mathrm{~W}$, in that case. Fig. 8 shows the distribution of this power on the contact area. It worth noting that since the steady state rolling velocity $V$, is in the $X$ direction we can sum the dissipation along this direction and plot the dissipated power $P_{l}$ per unit of $Y$ direction length. $P_{l}$ is directly related to the wear depth rate by Archard's law. Considering the $K / H V$ wear factor, the Archard's law enables to evaluate the depth rate $\dot{u}$, which will have the same aspect than $P_{l}$ along $y$ axis. Assuming linearity for a small time, one can
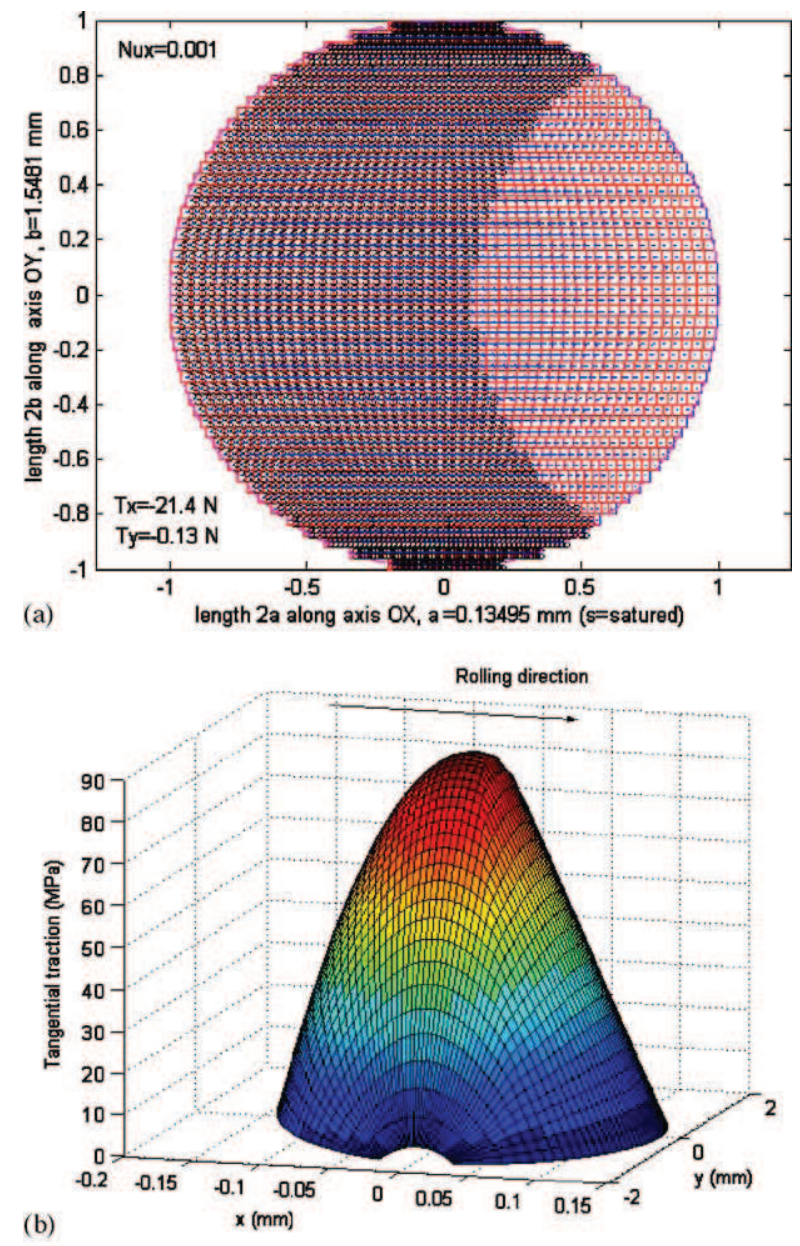

(b)

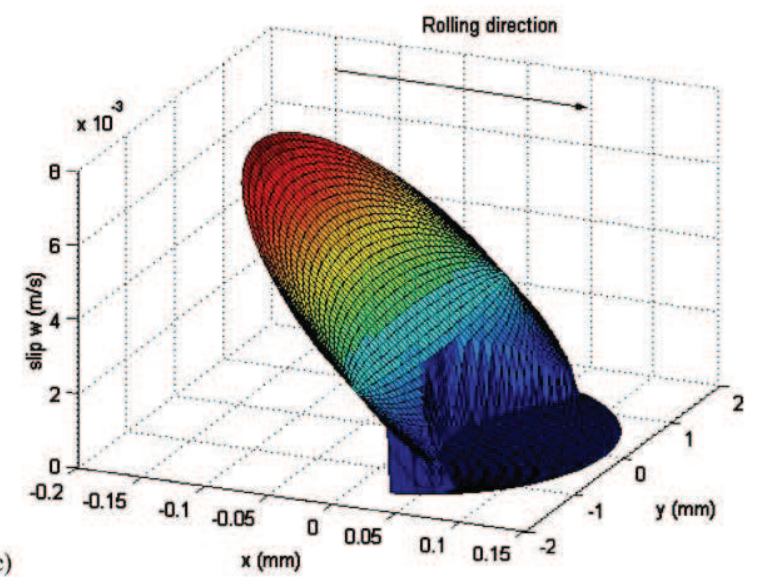

Fig. 7. (a) Dimensionless area with saturated element are marked with (s); (b) traction $\tau_{x}$; (c) slip $w_{x}$ in the contact area for the mean rolling problem.

identify the $K / H V$ factor from comparing depth $u_{\text {measured with }}$ depth $u_{\text {calculated }}$. Unfortunately, this test gives large dispersion on the identified factor. In the next section, we will see that even if the factor $K / H V$ is representative of the two bodies in contact, dispersion of test parameters can strongly affect the dissipated power and only a probabilistic approach can lead to an accurate identification of the wear law parameters. 

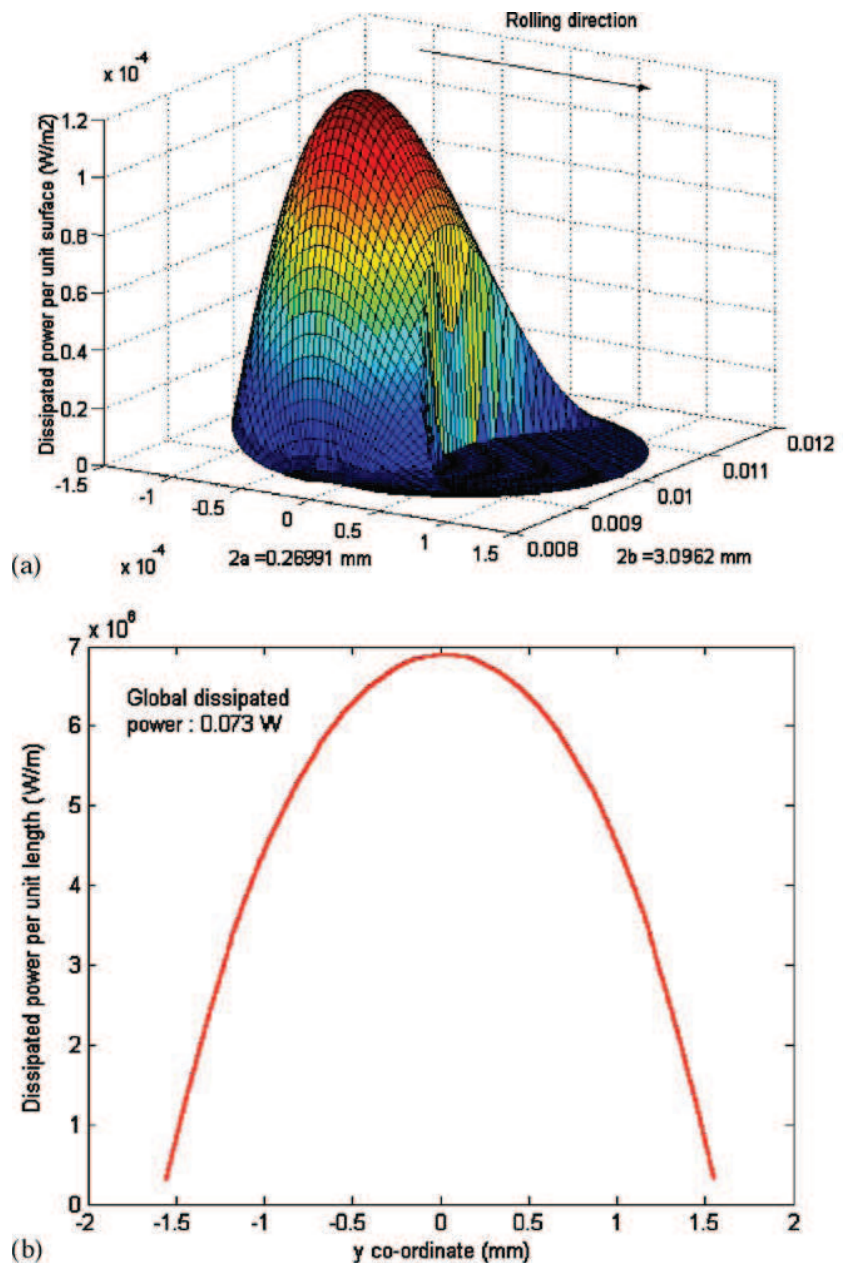

Fig. 8. (a) Dissipated power distribution on the contact area (right), (b) $\mathrm{P}_{l}$ vs. $y$ co-ordinate (left).

\section{Probabilistic approach of rolling contact}

To manage the probabilistic approach, the description of the dispersion of each parameter must be described by a probabilistic density function. In our case, parameters are normal load $N$, friction ratio $\mu$, longitudinal creep and angular defect between the roller axis and the cylinder axis. These parameters have very different origins and it is logical to consider that they are mutually independent. For each parameter, pdf allows numerous realisations of the random variable. The simulation presented in the previous section can be managed for each realisation. The construction of the probabilistic density function is briefly summarised below.

\subsection{Maximum entropy principle}

Considering a real-valued random variable $X$, associated with the probabilistic density function (pdf for short) $p_{X}(x)$, one can define the entropy by Eq. (13).

$S(X)=-\int_{[-\infty,+\infty]} p_{X}(x) \log \left(p_{X}(x)\right) \mathrm{d} x$
The maximum entropy principle (see [12-14] for applications) is a tool that allows the estimation of the pdf to be constructed by searching the maximum of $S(X)$ under the only available information. For example, the support of the pdf, the mean value, the standard deviation or higher moments. A Lagrange multiplier $\lambda_{i}$ will be associated to each constraint defined by the available information. These constraints are written in the Eq. (14) form.

$$
\begin{aligned}
E\left\{g_{i}(X)\right\} & =\int_{[-\infty,+\infty]} g i(x) p_{X}(x) \mathrm{d} x \\
& =f_{i}, \quad i=1, \ldots, m
\end{aligned}
$$

$f_{i}$ and $g_{i}(X)$ are given functions and are related to the means of $f_{i}$. For instance, if $g_{i}(x)=x, f_{i}$ is the mean value of $X$. It can be shown that the multipliers $X_{i}$ are obtained by minimising the strictly convex function $H\left(\lambda_{i}\right)$ defined by Eq. (15).

$$
\begin{aligned}
H\left(\lambda_{0}, \lambda_{1}, \ldots, \lambda_{m}\right)= & \lambda_{0}+\sum_{i=1}^{m} f_{i} \lambda_{i}+\int_{-\infty}^{\infty} 1_{[a, b]}(x) \\
& \times \exp \left(-\lambda_{0}-\sum_{i=1}^{m} \lambda_{i} g_{i}(x)\right) \mathrm{d} x
\end{aligned}
$$

$[a, b]$ is the support of the pdf of random variable $X$ and $l_{[a, b]}(x)$ the function equal to 1 if $x$ belongs to $[a, b]$ and 0 elsewhere. The pdf expression is then given by Eq. (16).

$p_{x}(x)=1_{[a, b]} \exp \left(-\lambda_{0}-\sum_{i=1}^{m} \lambda_{i} g_{i}(x)\right)$

Well known case of a random variable defined on the real line, with given mean value and standard deviation leads to a Gaussian law. In the next paragraph, we will construct the pdf associated to the random parameters for the wear problem.

\subsection{Probability density functions for the random parameters}

In the first section, it has been shown that loading fluctuation occurs during the wear test. The mean value of the normal load $N$ is denoted $m_{N}$ and equals $307 \mathrm{~N}$ for our case. Dispersion is characterised by the standard deviation $\sigma_{N}$ during the test which is equal to $32 \mathrm{~N}$. Of course $N$ cannot take negative values but no maximum values can arbitrarily be defined. Finally, the available information known to construct the pdf $P_{N}(n)$ is resumed in Eq. (17).

$\left\{\begin{array}{l}1=\int_{0}^{\infty} p_{N}(n) \mathrm{d} n \\ m_{N}=\int_{0}^{\infty} n p_{N}(n) \mathrm{d} n \\ \sigma_{N}^{2}+m_{N}^{2}=\int_{0}^{1} n^{2} p_{N}(n) \mathrm{d} n\end{array}\right.$

Minimising $H\left(\lambda_{N 0}, \lambda_{N 1}, \lambda_{N 2}\right)$ yields to $\lambda_{N 0}=50.40$, $\lambda_{N 1}=-0.299, \lambda_{N 2}=4.8810^{-4}$. With these three values, one 

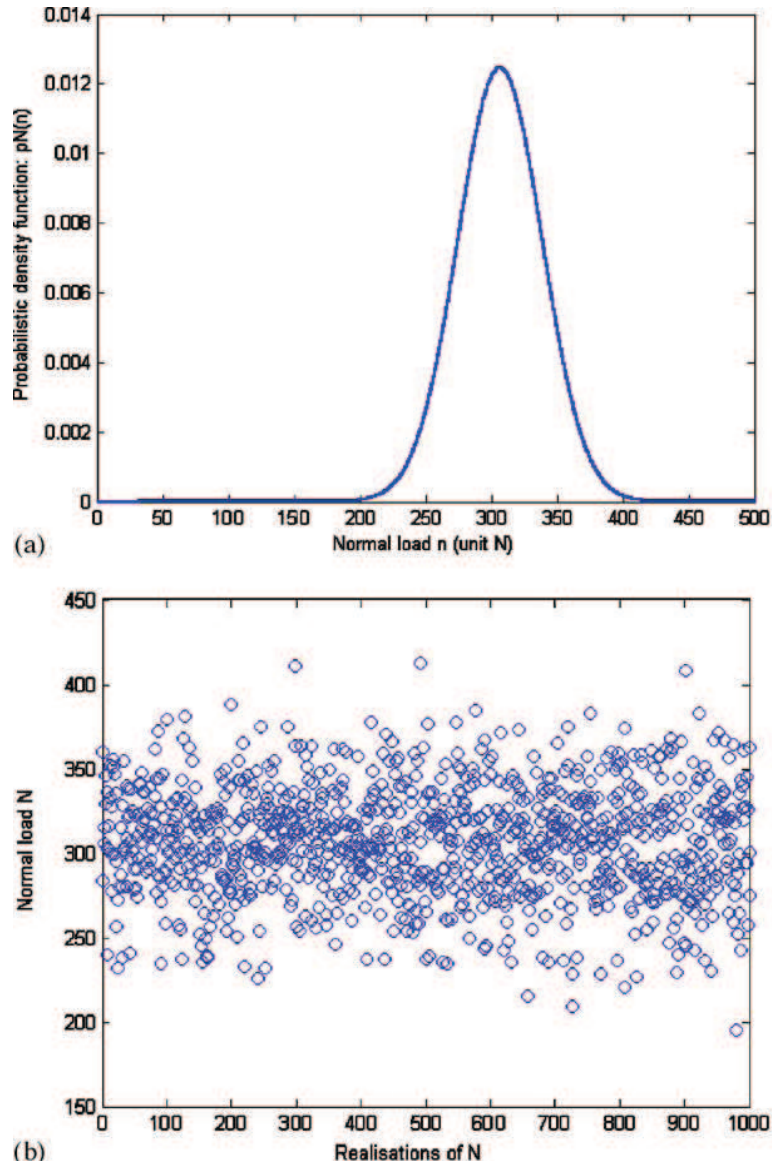

Fig. 9. (a) Probabilistic density function for load $N$, (b) 1000 realisations of random variable $N$ following the pdf.

can plot pdf $p_{N}(n)$ of the random variable $N$ (Fig. 9). For large values of $n$, this pdf looks like the Gaussian function but is strictly equal to 0 for negative values of $n$.

The random friction ratio $\mu$ can identically be treated because experimental measurements allow $m_{\mu}=0.1$ and $\sigma_{\mu}=0.02$ to be statistically estimated. Considering the longitudinal creep $v_{x}$, the treatment is slightly different because $v_{x}$ can only take values between 0 and 1 . The available information is then $m_{v_{x}}=0.001$ and $\sigma_{v_{x}}=0.0004$ and leads to the constraints of Eq. (18).

$$
\left\{\begin{array}{l}
1=\int_{0}^{1} p_{v_{x}}(u) \mathrm{d} u \\
m_{v_{x}}=\int_{0}^{1} u p_{v_{x}}(u) \mathrm{d} u \\
\sigma_{v_{x}}^{2}+m_{v_{x}}^{2}=\int_{0}^{1} u^{2} p_{v_{x}}(u) \mathrm{d} u
\end{array}\right.
$$

Minimising $H\left(\lambda_{v_{x}, 0}, \lambda_{v_{x}, 1}, \lambda_{v_{x}, 2}\right)$ yields to: $\lambda_{v_{x} 0}=-3.982$, $\lambda v_{x} 1=-5.86110^{3}, \lambda_{v_{x} 2}=2.95710^{6}$.

Other dispersion may occur because of the angular oscillation of the rolling body (Fig. 10a). Standard value for the amplitude $\gamma_{\max }$ of the oscillation is about $0.002 \mathrm{rad}$. Any value of $\gamma$ can be reached in the range $[-2 / 1000,2 / 1000]$ but
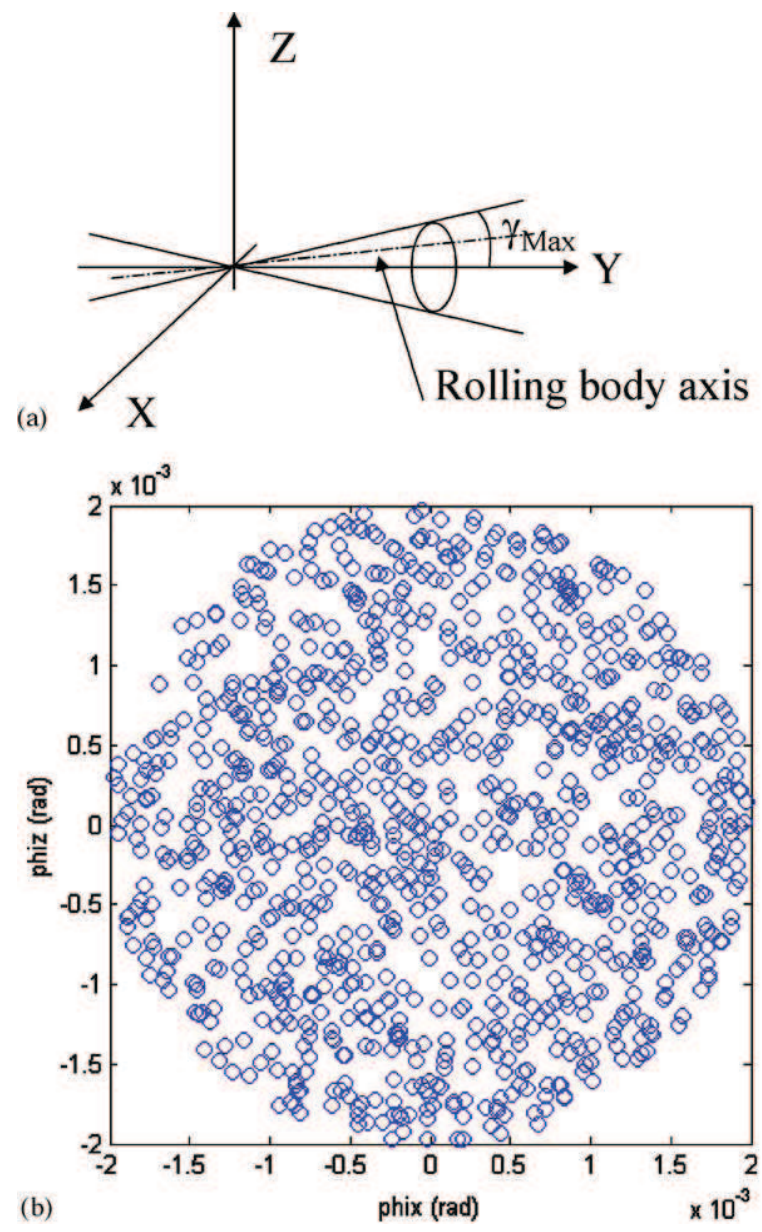

Fig. 10. (a) Maximum angular position for the rolling boy axis, (b) displays realisations of the vector-valued random variable $\left(\varphi_{x}, \varphi_{z}\right)$ following a uniform probabilistic density function.

every angular position can also be reached. Consequently the pdf of the random variable $\left(\varphi_{x}, \varphi_{z}\right)$ is uniform on the disc $K$ limited by Eq. (19).

$\varphi_{x}^{2}+\varphi_{z}^{2}=\gamma_{\max }^{2}$

The maximum entropy principle enables us to determine the pdf associated to this parameter. This pdf writes as shown on Eq. (20).

$p_{\left(\varphi_{x}, \varphi_{z}\right)}\left(\xi_{x}, \xi_{z}\right)=1_{k}\left(\xi_{x}, \xi_{z}\right) \frac{1}{\pi \gamma_{\max }^{2}} \quad$ with $\left(\xi_{x}, \xi_{z}\right) \in R^{2}$

Fig. 10b displays realisations of the vector-valued random variable $\left(\varphi_{x}, \varphi_{z}\right)$ from Eq. (20), it can be deduced that the random transversal creep and the random spin have zero mean values but the pdf is not uniform (Fig. 11). Standard deviations are respectively equal to $0.001 \mathrm{rad}$ and $0.05 \mathrm{rad} / \mathrm{m}$. This means that a uniform pdf on a disc does not yield a uniform distribution in the Cartesian co-ordinates of the position of a realisation of these co-ordinates. 


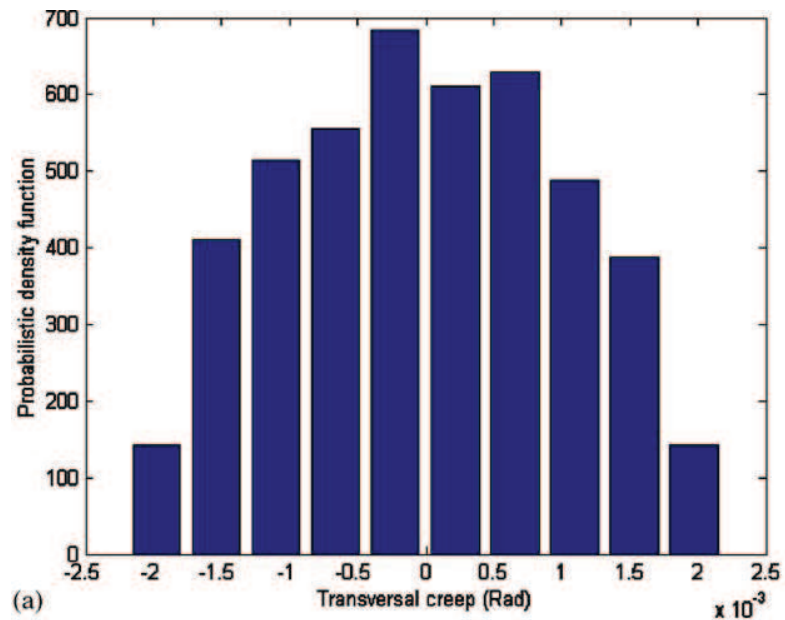

(a)

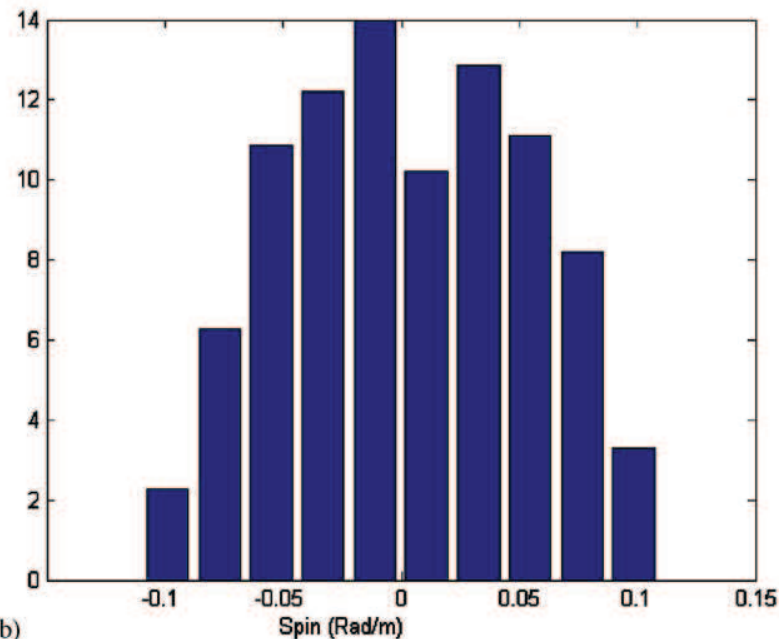

(b)

Fig. 11. Bar representation of pdf distribution of the random transversal creep (a), and the random spin (b), constructed with the realisations display in Fig. 10b.

\subsection{Probabilistic modelling of dissipation}

It should be noted that load $N$, creep $v_{x}, v_{y}$ and $\phi$ are global parameters but that friction ratio $\mu$ is a local one. During one of the 1000 calculations, for a $50 \times 50$ spatial discretisation of the contact area, 2500 realisations of the random variable $\mu$ are performed. One presents a typical result of one resolution of the steady state rolling problem using a probabilistic description of friction ratio $\mu$. As shown in Fig. 12, this produces a quite "chaotic" traction and slip distribution in the contact area that is certainly more representative of the real distribution.

It is still possible to sum the dissipated power along the rolling direction to have an evaluation of the dissipated power per unit length $P_{l}$. In Fig. 13, we can see that the distribution of dissipated power per unit length is more similar of experimental wear profiles than the smooth chart of Fig. 8b. For this realisation, the global dissipation, as well as the mean distribution of $P_{l}$, are a little smaller than the mean value obtained by the determinist simulation of the mean problem.
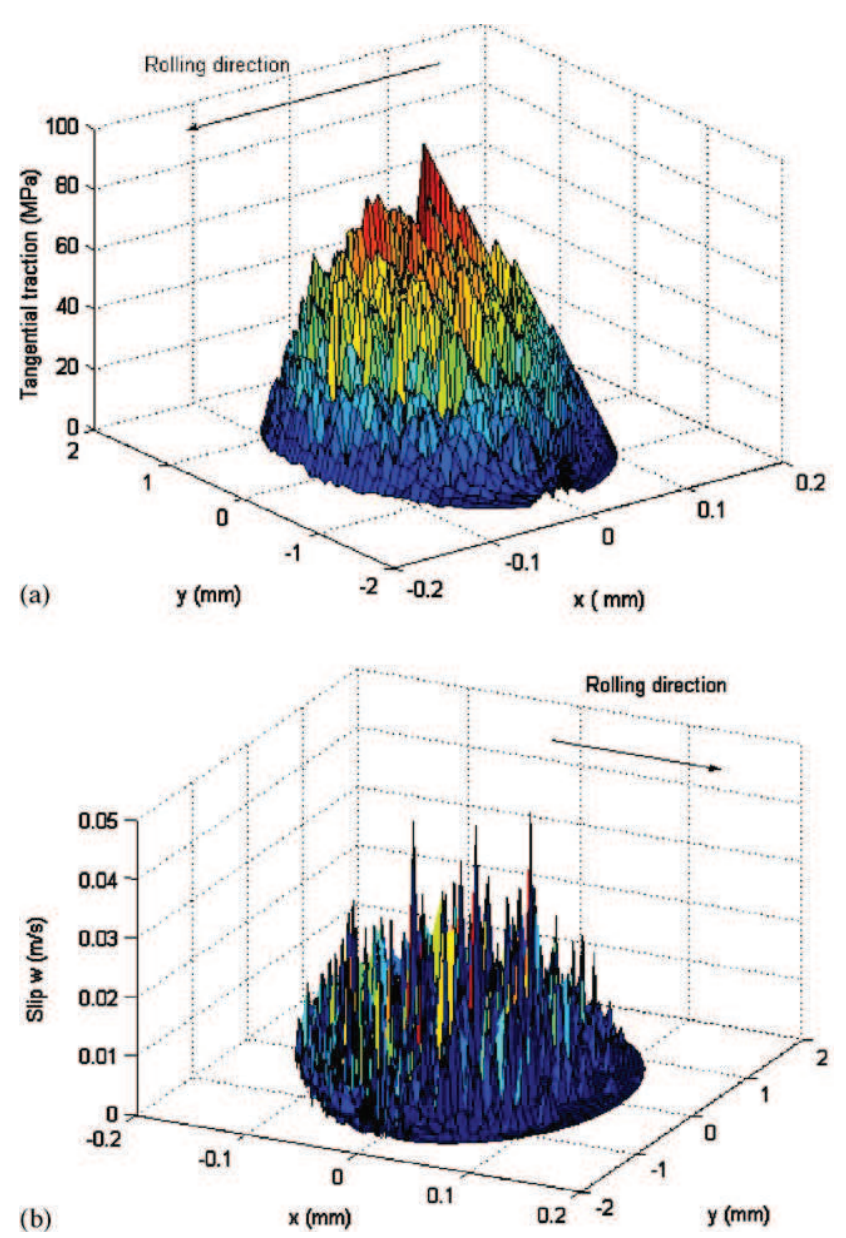

Fig. 12. 'Chaotic' distribution of traction (a), and slip (b) issued of a Fastsim simulation with probabilistic modelling of the friction ratio.

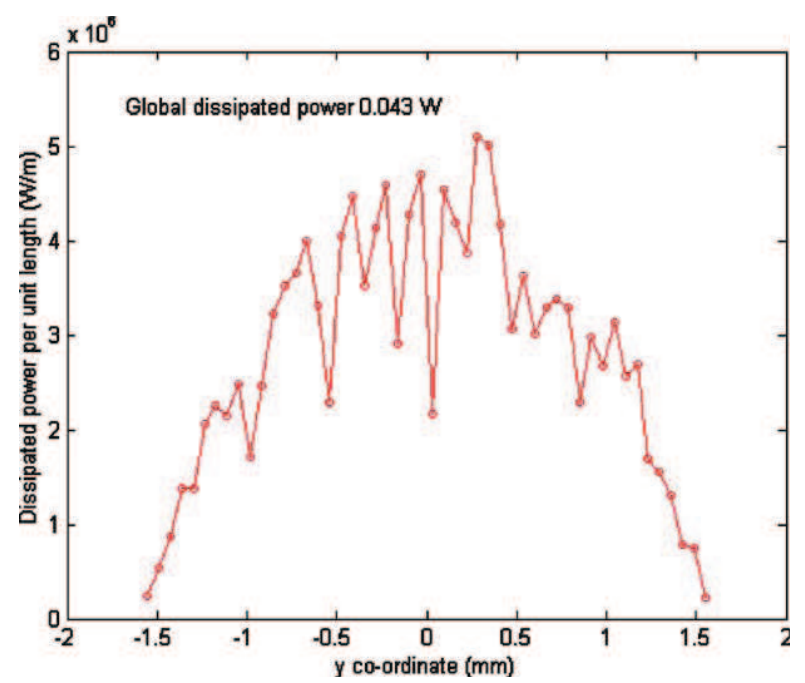

Fig. 13. Dissipated power per unit length issued from probabilistic description of the friction ratio over the contact area. 
This can be due to a lower normal load or a small longitudinal creep of this specific realisation. This way to treat the contact problem, will lead to the definition of the confidence region of the solution with respect to the measured dispersion of the probabilistic parameters of the mechanical problem. We now present the results of this approach.

\section{Results and discussion}

In this section, we will examine the result of a Monte-Carlo simulation [15] of the steady state rolling contact problem. As presented above, we carried 1000 numerical simulations with Fastsim: one presents the convergence of the description and show the construction of a probability density function for the random dissipated power which is the result of the simulation. An iterative method could be used in order to follow the wear evolution. Presently, we limit the development to the random dissipated power per unit length.

\subsection{Monte-Carlo numerical simulation: convergence}

Convergence of the Monte-Carlo numerical simulation can be shown by the calculation of the mean value and the standard deviation of the random global dissipated power. In Fig. 14, both terms are plotted versus the number of realisations managed. We can see that convergence is managed for 300 realisations. The mean value of the dissipated power appears to be equal to $0.127 \mathrm{~W}$ which is clearly different from the dissipated power value obtained by solving the mean problem. Let us recall that the dissipated power found was equal to $0.073 \mathrm{~W}$ which is $42 \%$ less than the mean value of the probabilistic approach. This difference is due to transversal creep $v_{y}$ and spin $\phi$. Even if the mean values of these two terms are equal to zero, the non-zero values that can be positive or negative during the test, all lead to a higher dissipation than the mean value.

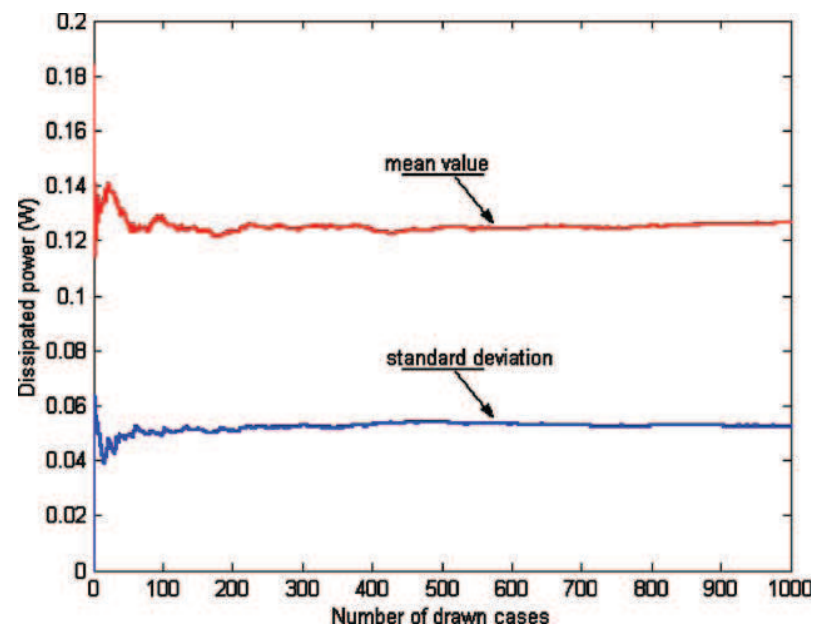

Fig. 14. Evolution of mean value and standard deviation vs. the number of realisation.
This is not the case for other variables as the normal load $N$, for example; when $N$ is lower than the mean value, the dissipation is also lower. This is also the case for friction ratio or longitudinal creep.

\subsection{Mean dissipation and confidence range}

The small number of realisation (about 300) is enough to obtain convergence for first and second order moments of the random dissipated power $P_{\mathrm{d}}$, but is not enough to obtain convergence for the higher moments. With 1000 realisations one can construct a estimation of the probability density function of the random variable $P_{\mathrm{d}}$.

Fig. 15a shows a bar chart built from the choice of a number of class (here 30 classes) in the interval $\left[P_{\mathrm{d}}^{\mathrm{Min}}, P_{\mathrm{d}}^{\mathrm{Max}}\right]$. $P_{\mathrm{d}}^{\mathrm{Min}}$ and $P_{\mathrm{d}}^{\mathrm{Max}}$ are minimum and maximum values taken by $P_{\mathrm{d}}$ over the 1000 realisations. We can count the number of realisations that give a $P_{\mathrm{d}}$ value for each class. This number reported to the 1000 values enables the determination of the probability to have a realisation belonging to this class.
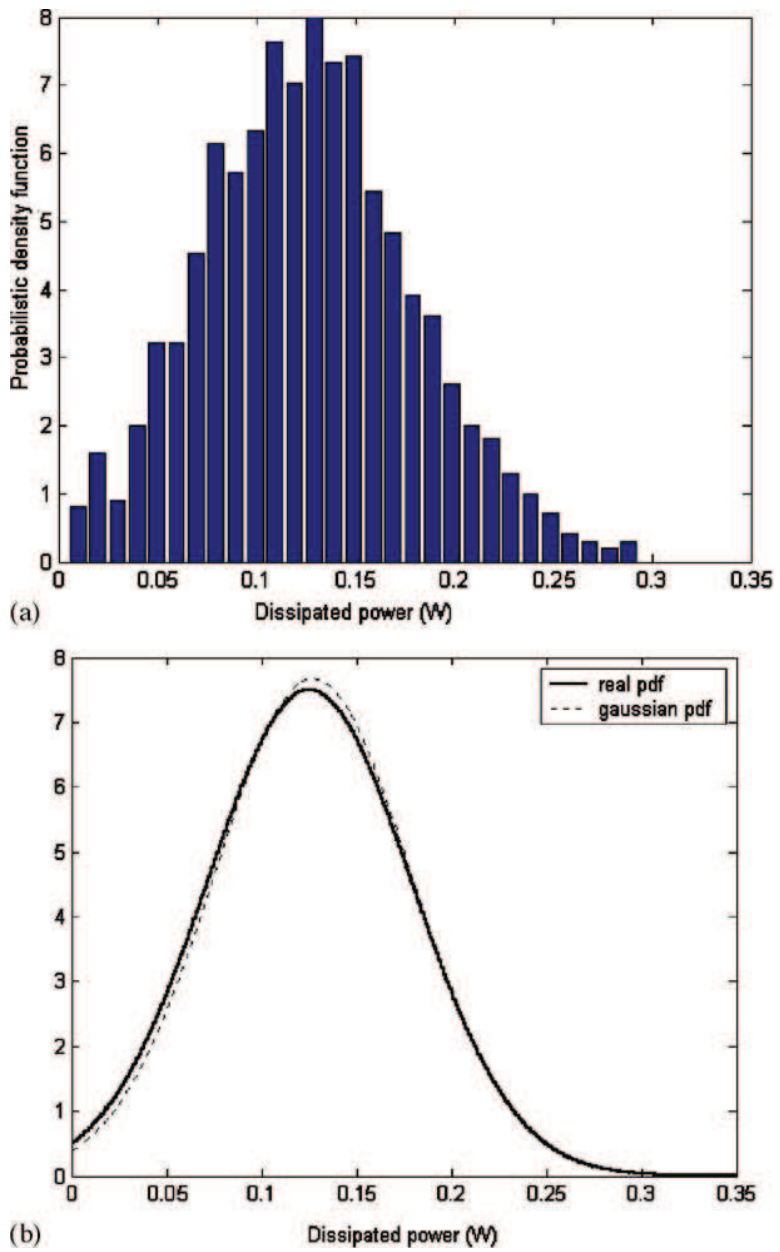

Fig. 15. Probability density function of the dissipated power (a) representation from Monte-Carlo simulation with 30 class (b) identified from the mean value and standard deviation calculated from the Monte-Carlo simulation. 


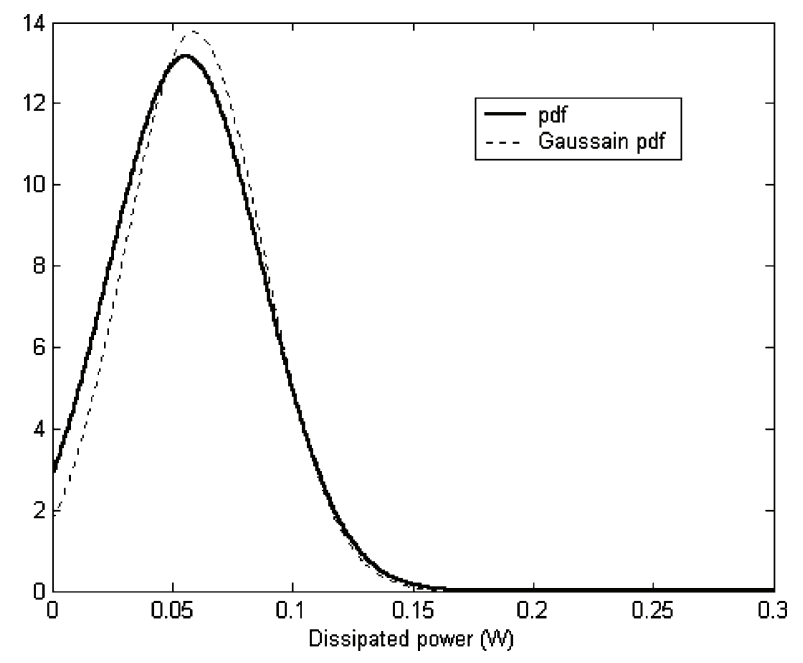

Fig. 16. Dissipated power pdf identified from the Monte-Carlo simulation managed with no transversal creep and spin.

When the number of realisation grows up, this chart becomes a continuous probabilistic density function (Fig. 15b). One can see the difference with the Gaussian function that have the same mean value and same standard deviation than the probabilistic variable $P_{\mathrm{d}}$.

As a comparison, we show on Fig. 16, the density function obtained by the same Monte-Carlo approach without taking into account the influence of $v_{y}$ and $\phi$ dispersion. In that case, the mean value of $P_{\mathrm{d}}$ is $0.068 \mathrm{~W}$ which the same order of magnitude than the mean model value.

Considering the chart of Fig. 15, it is possible to define a confidence region where probability to find a realisation is higher than $P_{\mathrm{c}}$ (for example, $P_{\mathrm{c}}=95 \%$ ). The upper and lower bounds of this confidence region are obtained by Eq. (21).

$p_{\mathrm{d}}^{+}=\zeta\left(1-P_{\mathrm{c}}\right)$ and $P_{\mathrm{d}}^{-}=\zeta\left(P_{\mathrm{c}}\right)$

$\zeta(p)$ is the fractile defined for a probabilistic variable $X$ with a distribution function $F_{x}(x)$, by:

$\zeta(p)=\inf \left\{x, F_{X}(x)<p\right\}$

This can simply be done by counting the realisations ordered by dissipated power values until the density function reaches $P_{\mathrm{c}}$ for the upper bound and $\left(1-P_{\mathrm{c}}\right)$ for the lower bound. In the case of the pdf of dissipated power presented in Fig. 15 , the boundaries are $P_{\mathrm{d}}^{+}=0.012 \mathrm{~W}$ and $P_{\mathrm{d}}^{-}=0.29 \mathrm{~W}$. It appears that the mean value $0.127 \mathrm{~W}$ is included but not centred (centred value should be $0.152 \mathrm{~W}$ ) on the confidence interval. Finally, if we consider the confidence domain amplitude in comparison with the value obtained by solving the mean problem, it appear that dissipated power can reach two or three times the expected value classically calculated by a mean determinist model. This conclusion is sufficient to justify the use of the probabilistic approach for contact problem.

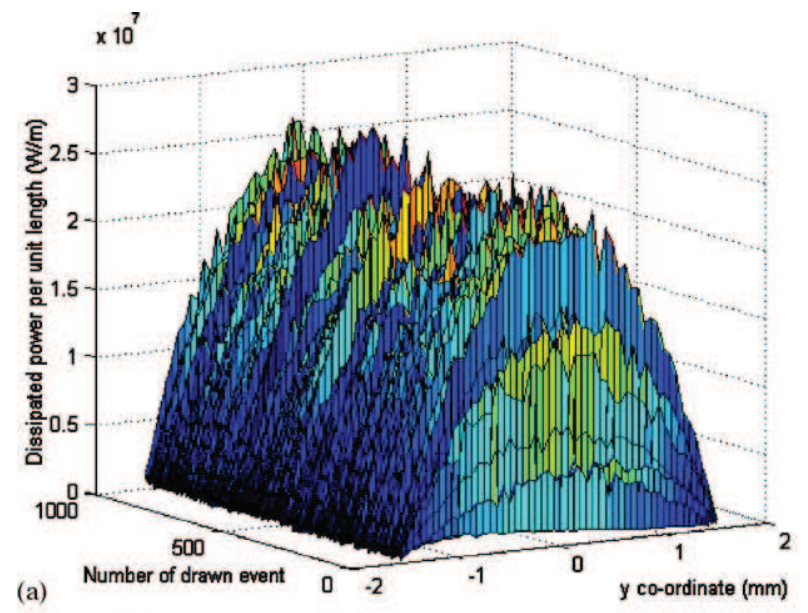

(a)

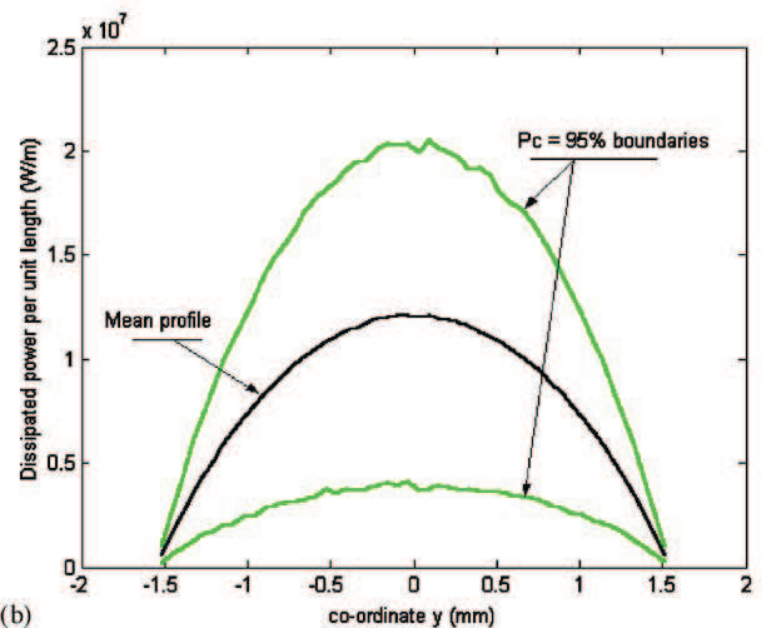

Fig. 17. Dissipated power per unit length. (a) All probabilistic chart, (b) mean distribution of $P_{l}$ and minimal and maximal boundaries of the confidence region for a confidence probability $P_{\mathrm{c}}=95 \%$.

\subsection{Mean wear: dissipation per unit length}

In this last section, we will examine the dissipated power per unit length $P_{l}(y)$ which is associated to the wear depth. Fig. 17a shows all the distributions of $P_{l}(y)$ for the 1000 realisations. The statistical treatment presented above for the scalar variable $P_{\mathrm{d}}$ can be done for each discrete value $P_{l}\left(y_{i}\right)$ and gives the mean profile and the confidence region for $95 \%$ viability. It worth noting that, in that case, the confidence region is nearly centred on the mean profile.

This can seem contradictory with the conclusion on $P_{\mathrm{d}}$ but one must notice that in all realisation we have a large number of case where spin values leads to non symmetric $P_{l}(y)$. All those cases give high values of $P_{\mathrm{d}}$ and the confidence region is larger over the mean value of this variable. Fig. 17b clearly shows an important dispersion that lead to a large uncertainties of the wear evolution. This explains that "identical" combination of the wear apparatus lead to very different evolution during the test. 
Identification of an Archard's wear factor can easily be done from a mean measured profile. The measured dispersion on wear profiles can help to concluded on the nature of the factor ' $K$ ' whether it is a determinist coefficient or also a probabilistic one. Considering microscopic approach [16] explains that ' $K$ ' is a function of material asperity's geometry, the question can be studied.

\section{Conclusions}

On this simple study, we showed how a probabilistic approach can lead to evaluation of the wear dispersion observed during wear tests. A simplified contact model is used in order to managed a large number of simulation an make statistical analysis of the dissipated power.

This work is the first step to the realisation of a wear simulation tool, and the comparison between dissipated power per unit length and wear depth profiles enables a simple wear law identification. Considering the dispersions on experimental results this seems to be the only possible approach.

It is worth noting that if wear occurs during a long time, contact geometry might be changed and the radius of contact profiles have to be updated during simulation. Such a scheme has been used successfully in [17], [18] or [1]. Recently [19] have proposed a semi-hertzian approach in case of important geometrical modification which will be used in the next step of this work.

\section{Acknowledgement}

Sidel provides financial support to this study.

\section{References}

[1] L. Chevalier, H. Chollet, Endommagement des pistes de roulement, Mech. Ind. 1 (2000) 593-602.
[2] J.F. Archard, J. Appl. Phys. 24 (1953) 981-988

[3] Li Zi-Li, J.J. Kalker, Simulation of Severe Wheel-Rail Wear, Delft University, 1996.

[4] A. Magnée, Modelization of damage by abrasion, Wear 162-164 (1993) 848-855.

[5] A.E.H Love, A Treatise on the Theory of Elasticity, fourth ed., Cambridge University press, 1926.

[6] K.L. Johnson, Contact Mechanics, Cambridge University Press, 1985.

[7] J.J. Kalker, A fast algorithm for the simplified theory of rolling contact, Program FASTSIM, Vehicle systems dynamics, vol. 111-113, SWETS \& ZEITLINGER B.V., LISSE, 1982.

[8] E. Felder, Mécanismes physiques et modélisation mécanique du frottement entre corps solides, Mech. Ind. (2000) 555-561.

[9] V.M. Alexandrov, Asymptotic methods in contact mechanics, Math Comput. Model. 128 (n4-n8) (1998) 29-36.

[10] A.M. Habraken, S. Cescotto, Contact between deformable solids: the fully coupled approach, Math Comput. Model. 28 (n4-n8) (1998) 53-170.

[11] Z.L. Li, J.J. Kalker, Adaptative solution of rolling contact using boundary element method, in: Proceedings of the 20th World Conference on the Boundary Element Method, Orlando, FL, USA, 1998.

[12] E.T. Jaynes, Information theory and statistical mechanics, Phys. Rev. 106 (4) (1957) 620-630, and 108 (2) 171-190.

[13] J.N. Kapur, H.K. Kesavan, Entropy Optimisation Principle with Applications, Academic Press, San Diego, 1992.

[14] C. Soize, Maximum entropy approach for modelling random uncertainties in transient elastodynamics, J. Acoust. Soc. Am. 109 (5) (2001).

[15] M.H. Kalos, P.A. Whitlock, Monte-Carlo Methods Volume 1: Bascs, Wiley, Chistester, 1992.

[16] D. François, A. Pineau, A. Zaoui, Comportement mécanique des matériaux, tome 2, Hermés 401-450 (1993) 159-166.

[17] Z.L. Li, J.J. Kalker, Computation of wheel-rail conformal contact, in: Proceedings of the Fourth World Congress on Computational Mechanics, BuenosAires, July, 1998.

[18] W. Kik, J. Piotrowski, A fast approximate method to calculate normal load at contact between wheel and rail, and creep forces during rolling, in: Proceedings of the Second Mini-Conference on Contact Mechanics and Wear of Rail/Wheel Systems, Warsaw Technical University, Budapest, July, 1996, pp. 29-31.

[19] J.B. Ayasse, H. Chollet, 'Determination of the wheel rail contact patch in semi-Hertzian conditions, Vehicle Syst. Dynam. (2002). 